\title{
Viewpoint
}

\section{Simplest Water Cluster Leaves Behind its Spectral Fingerprint}

\author{
Richard J. Saykally \\ Department of Chemistry, University of California, Berkeley, CA 94720, USA \\ Published February 25, 2013
}

Water dimers have been detected in room-temperature water vapor, a key step toward understanding their effect on solar absorption and chemistry in the atmosphere.

Subject Areas: Chemical Physics, Geophysics

\author{
A Viewpoint on: \\ Water Dimer Rotationally Resolved Millimeter-Wave Spectrum Observation at Room Temperature \\ M. Yu. Tretyakov, E. A. Serov, M. A. Koshelev, V. V. Parshin, and A. F. Krupnov \\ Phys. Rev. Lett. 110, 093001 (2013) - Published February 25, 2013
}

Water is the third most abundant molecule in the atmosphere and the principal absorber of both incoming sunlight and reradiated blackbody radiation. Yet models of atmospheric absorption that only take into account the water molecule's well-known rotational and vibrationrotational transitions don't match up with measurements of the atmosphere's absorption spectrum [1]. One possibility is that water dimers - two water molecules held together by a hydrogen bond - and larger water clusters act as sources of excess absorption. The idea has been intensely debated for over four decades, with recent work suggesting that water dimers are involved in both the radiation balance and chemistry of the atmosphere 2 . Unfortunately, definitive detection of water dimers in the atmosphere has remained elusive: several promising reports later proved to be either ambiguous or incorrect [3, and laboratory detection schemes that were successful were performed under conditions unlike those in the atmosphere.

In Physical Review Letters, Mikhail Tretyakov and colleagues at the Russian Academy of Sciences in Nizhny Novgorod present a vital and long-sought first step towards detecting dimers in the atmosphere 4. Using a highly sensitive spectrometer technique that they developed, the team measured the absorption of millimeterwave radiation in water vapor at room temperature and equilibrium conditions, identifying four peaks associated with the rotational motion of the dimers. The ability to detect the dimers' "spectral fingerprint" under environmentally relevant conditions could provide scientists a way to directly measure their abundance in various parts of the atmosphere.

Nearly all the water vapor in the atmosphere lies in the troposphere - the layer extending roughly 20 kilometers from Earth's surface. Dimers make up less than one percent of this water vapor, which makes it difficult to see them compared to single (monomer) water molecules, particularly in the visible and infrared por-

DOI: $10.1103 /$ Physics.6.22

URL: http://link.aps.org/doi/10.1103/Physics.6.22 tion of the spectrum, where the light energy is sufficient to destroy the dimers.

Experiment and theory suggest that it would be easier to distinguish the spectral contribution from dimers with millimeter-wave light, but conventional spectrometers aren't sufficiently sensitive at these frequencies. For several years, the Russian group has been developing the technology to perform millimeter-wave spectroscopy on molecules under atmospheric conditions. The heart of their spectrometer is a resonator: a cavity with mirrors on either end that optimally selects for a particular frequency of light depending on the separation between the mirrors. The sharpness, or "quality factor," of this resonance frequency depends on how much light is absorbed by the gas in the cavity. The authors are therefore able to map out the absorption of a gas or vapor in the cavity by stepping the spacing between the mirrors and measuring the quality factor at each point.

Based on accurate calculations 5] and spectral measurements of water dimers made in cold molecular beams, the authors were able to predict where the dimers' rotational transitions would lie in the region 100-150 gigahertz $(\mathrm{GHz})$ 6. In their measured absorption spectrum of water vapor at $23^{\circ} \mathrm{C}$ and 0.022 atmospheres (atm) of pressure (Fig. 1), they identified a series of four absorption peaks as originating in increasingly energetic rotational states of the water dimers. If the authors' measurement is confirmed by other experiments, it will constitute the first detection of water dimers under ambient temperature equilibrium conditions.

The contrast between the discrete features in the dimer spectrum and the underlying continuum absorption is low, but the fact that four separate features can be observed in exactly the predicted positions lends confidence to the assignment. Moreover, Tretyakov et al. have carefully examined several crucial aspects of their experiments, and present additional evidence in favor of their conclusion. Based on their measurement, the authors es- 


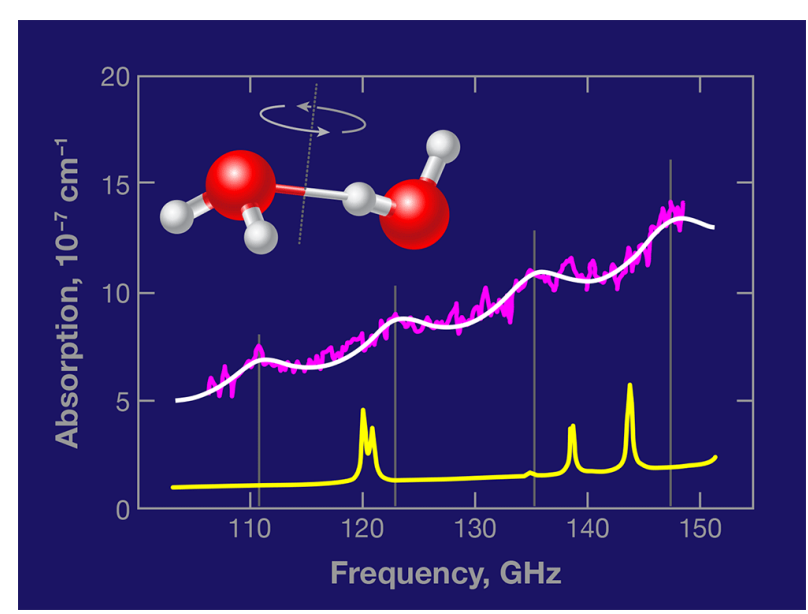

FIG. 1: High-resolution spectroscopy is allowing scientists to detect the presence of water dimers - the simplest cluster of water molecules (inset) - in a sample of water vapor. The pink line is the measured spectrum and the white line is the calculated dimer spectrum. (The monomer contribution -indicated by the yellow trace-has been subtracted.) The vertical bars show the positions of the dimer rotational lines. The spectrum was measured in water vapor in equilibrium at $23{ }^{\circ} \mathrm{C}$ and $0.022 \mathrm{~atm}$. (APS/Alan Stonebraker from Ref. 4])

timate that the relative abundance of the dimers in the equilibrium vapor is roughly $1 \times 10^{-3}$.

There is, however, one issue that remains incompletely addressed. The spectral lines measured by Tretyakov et al. are nearly four times broader than those predicted by calculations [6]. The authors argue that this discrepancy occurs because additional transitions - the same dimer rotational transitions, but occurring in lowenergy excited intermolecular vibrations (known as "hot bands") - are also allowed near the ground vibrational state dimer transitions. This could be correct, but it seems surprising to see these transitions occur with the same regular frequency shifts as the ground-state rotational transitions. The origin of the broadening needs to be clarified before the dimer spectrum can be used to quantitatively detect water dimers in the atmosphere. Moreover, the new experiment of Tretyakov et al. presents the opportunity to characterize the collisional broadening of the water dimer transitions, which would be of much intrinsic interest.

The study by Tretyakov et al. might also provide new impetus to reevaluate the interpretation of earlier experiments. In 1969, Bohlander et al. reported the still unconfirmed detection of water dimers made under similar equilibrium conditions, but in the far infrared [7. The authors of this study used a very long pass absorption cell with a far-infrared absorption spectrometer to observe a weak feature near $23 \mathrm{~cm}^{-1}(690 \mathrm{GHz})$. The temperature dependence of this feature provided evidence that it originated from the dimer, rather than the monomer, but although other researchers repeated the experiment, they were never able to reproduce the results. In 1989,

DOI: $10.1103 /$ Physics.6.22

URL: http://link.aps.org/doi/10.1103/Physics.6.22 an independent group (including myself) [8] observed a number of water dimer rotational-tunneling transitions in this same spectral region at very high spectral resolution using a tunable far-infrared sideband laser and a planar supersonic jet that produced the water dimers at a temperature of $4 \mathrm{~K}$. Several of these transitions were found to occur within the width of the broad lines reported by Bohlander et al., a correspondence that should be investigated more thoroughly. Furthermore, our group also reported [9] the measurement of several vibration-rotationtunneling transitions in the argon- $\mathrm{H}_{2} \mathrm{O}$ complex in this same spectral range. Finally, satellite measurements of water vapor emission in the atmosphere by microwave limb sounding instruments have been made in nearly the same spectral range as used by Tretyakov et al. and could be employed to search for the same water dimer transitions [10]. Taken together, these previous studies and the new work from Tretyakov et al. offer encouraging signs that new experimental techniques will bring closure to the important challenge of understanding the absorption spectrum of the Earth and the contribution of water to that absorption.

\section{References}

[1] N. Goldman, C. Leforestier, and R. J. Saykally, "Water Dimers in the Atmosphere II: Results from the VRT(ASPW)III Potential Surface," J. Phys. Chem. A 108, 787 (2004).

[2] V. Vaida, "Perspective: Water Cluster Mediated Atmospheric Chemistry," J. Chem. Phys. 135, 020901 (2011).

[3] A. J. L. Shillings, S. M. Ball, M. J. Barber, J. Tennyson, and R. L. Jones, "An Upper Limit for Water Dimer Absorption in the $750 \mathrm{~nm}$ Spectral Region and a Revised Water Line List," Atmos. Chem. Phys. 11, 4273 (2011).

[4] M. Y. Tretyakov, E. A. Serov, M. A. Koshelev, V. V. Parshin, and A. F. Krupnov, "Water Dimer Rotationally Resolved Millimeter-Wave Spectrum Observation at Room Temperature," Phys. Rev. Lett. 110, 093001 (2013).

[5] Y. Scribano and C. Leforestier, "Contribution of Water Dimer Absorption to the Millimeter and Far Infrared Atmospheric Water Continuum," J. Chem. Phys. 126, 234301 (2007).

[6] A. F. Krupnov, M. Yu. Tretyakov, and C. Leforestier, "Possibilities of the Observation of the Discrete Spectrum of the Water Dimer at Equilibrium in Millimeter-Wave Band," J. Quant. Spectrosc. Radiat. Transfer 110, 427 (2009).

[7] R. A. Bohlander, H. A. Gebbie, and G. W. F. Pardoe, "Absorption Spectrum of Water Vapor in the Region of $23 \mathrm{~cm}^{-1}$ At Low Temperatures," Nature 228, 157 (1970).

[8] K. L. Busarow, R. C. Cohen, G. A. Blake, K. B. Laughlin, Y. T. Lee, and R. J. Saykally, "Measurement of the Perpendicular Rotation-Tunneling Spectrum of the Water Dimer by Tunable Far Infrared Laser Spectroscopy in a Planar Supersonic Jet," J. Chem. Phys. 90, 3937 (1989).

[9] R. C. Cohen, K. L. Busarow, K. B. Laughlin, G. A. Blake, M. Havenith, Y. T. Lee, and R. J. Saykally, "Tunable Far Infrared Laser Spectroscopy of van der Waals Bonds: VibrationRotation-Tunneling Spectra of $\mathrm{Ar}-\mathrm{H}_{2} \mathrm{O}$," J. Chem. Phys. 89, 4494 (1988).

[10] H. C. Pumphrey and S. Buhler, "Instrumental and Spectral Parameters: Their Effect on and Measurement by Microwave Limb Sounding of the Atmosphere", J. Quant. Spectrosc. Radiati. Transfer 64, 421 (2000). 


\section{About the Author}

\section{Richard J. Saykally}

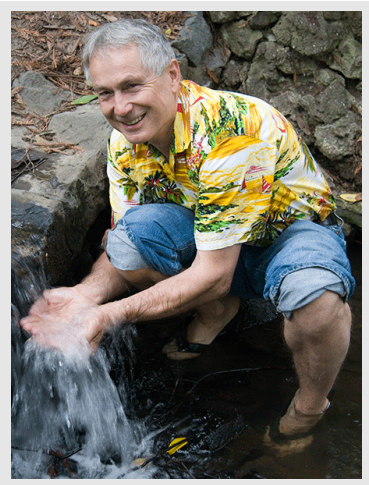

Richard Saykally received his Ph.D. from the University of Wisconsin, Madison, after which he took a postdoctoral appointment at the National Bureau of Standards (now NIST) in Boulder, Colorado. A professor at the University of California, Berkeley, since 1979, he is a coauthor of over 400 publications and the recipient of over 70 honors and awards from nine different countries. He is a member of the National Academy of Sciences and the American Academy of Arts and Sciences and a UC-Berkeley Distinguished Teacher. He has also been active at the national level in science education. Saykally currently holds The Class of 1932 Endowed Chair in the Department of Chemistry. 